\title{
ON THE SELF-INTERSECTIONS OF FOLIATION CYCLES
}

\author{
YOSHIHIKO MITSUMATSU \\ Dedicated to Professor Masahisa Adachi on his 60th birthday
}

\begin{abstract}
The existence of a transverse invariant measure imposes a strong restriction on the transverse complexity of a foliated manifold. The homological self-intersection of the corresponding foliation cycle measures the complexity around its support. In the present paper, the vanishing of the self-intersection is proven under some regularity condition on the measure.
\end{abstract}

\section{INTRODUCTION}

It is a basic problem for the theory of foliations to investigate how the transverse structures can be twisted. If a foliation has a foliation cycle, its dynamical behaviour becomes mild in some sense. In this paper, we study the homological self-intersection of foliation cycles which reflects such a phenomenon. For a foliation cycle $C$, a similar invariant $\chi_{\nu}(\mathscr{F})=[C] \cap e(\nu \mathscr{F})$ is considered, where $e(\nu \mathscr{F})$ denotes the euler class of the normal bundle $\nu \mathscr{F}$ to $\mathscr{F}$. The self-intersection $[C]^{2}$ has some relation to $\chi_{\nu}(\mathscr{F})$ as explained later. As for the estimate or the vanishing of $\chi_{\nu}(\mathscr{F})$, see [3 and 5].

The leaves of a foliation $\mathscr{F}$ fili up a foliated manifold $(M, \mathscr{F})$ without intersecting with each other. This suggests the vanishing of the self-intersection in many cases. In this paper a sufficient condition for the vanishing is given.

Throughout this paper, we assume that a foliated manifold $(M, \mathscr{F})$ is both tangentially and transversely oriented, $M$ and $\mathscr{F}$ are smooth, and $M$ is closed. Let $p, q$, and $n$ be $\operatorname{dim} \mathscr{F}, \operatorname{codim} \mathscr{F}$, and $\operatorname{dim} M$ respectively. The tangent (resp. normal) bundle to $\mathscr{F}$ is denoted by $\tau \mathscr{F}$ (resp. $\nu \mathscr{F}$ ). The foliation cycle (resp. the invariant measure) which corresponds to a transverse invariant measure $\mu$ (resp. the foliation cycle $C$ ) is denoted by $C=C_{\mu}$ (resp. $\mu=\mu_{C}$ ). Homology and cohomology always imply de Rham's ones. $\Omega^{*}$ denotes the de Rham cochain complex. For the fundamentals of foliation cycles, see [7].

The author is deeply grateful to Professor A. Hattori and Professor S. Morita for their advice and continuous encouragement.

Received by the editors March 12, 1990 and, in revised form, September 21, 1990.

1980 Mathematics Subject Classification (1985 Revision). Primary 57R20, 57R30; Secondary $28 \mathrm{D} 15$. 


\section{The fundamental Classes of COMPACt leaves}

First, we deal with the fundamental classes of compact leaves as a special case of foliation cycles. Through some examples, we make simple observations on their self-intersections. To begin with, we review the following two well-known propositions, first of which is known as the Bott vanishing theorem and is one of the starting points of this paper.

Proposition 1.1 [1]. The normal bundle $\nu \mathscr{F}$ admits a $G L^{+}(q, \mathbb{R})$-connection which is flat along the leaves of $\mathscr{F}$.

Such a connection is called a "Bott connection" or a "basic connection" and is not unique.

Proposition 1.2 (Milnor-Sullivan-Gromov-Smillie, $[4,6,2])$. Let $X$ be an oriented closed p-manifold and $\xi$ be a flat oriented $\mathbb{R}^{p}$-vector bundle over $X$. Then the inequality

$$
|\langle e(\xi),[X]\rangle| \leq 2^{-p}\|X\|
$$

holds, where $\|X\|$ denotes the Gromov invariant of $X$. Especially in the case of $p=2$, the original Milnor inequality has the expression

$$
|\langle e(\xi),[X]\rangle| \leq 2^{-1} \max \{0,-\chi(X)\},
$$

where $\chi(X)$ is the euler characteristic of $X$. 1.4 .

Combining these propositions and the next easy one, we obtain Proposition

Proposition 1.3. For a compact leaf $L$ of a foliated manifold $(M, \mathscr{F})$, the following relation

$$
[L] \cdot[L]=[L] \cap e(\mathscr{F}) \in H_{p-q}(M)
$$

holds. If $p=q$, of course the r.h.s. is nothing but the Kronecker product.

Proposition 1.4. Let $L$ be a compact leaf of a p-dimensional foliation $\mathscr{F}$ on a $2 p$-dimensional manifold $M$. Then we have

$$
|\langle e(\nu \mathscr{F}),[L]\rangle|=\left|[L]^{2}\right| \leq 2^{-p}\|L\| .
$$

Remark. Proposition 1.3 does not hold for general foliation cycles, e.g., if we take $2[L]$ instead of $[L]$, the 1.h.s. is multiplied by $2^{2}=4$, but the r.h.s. is multiplied by 2 .

Example 1.5. Let us consider a foliated $S^{2}$-bundle $\zeta: M^{4} \rightarrow \Sigma_{g}$ over a closed oriented surface $\Sigma_{g}$ of genus $g(g \geq 2)$. For a given integer $J$ such that $|J| \leq 2^{-1}\left|\chi\left(\Sigma_{g}\right)\right|=g-1$, we can construct a foliated $S^{2}$-bundle $\zeta$ which has a compact leaf with its self-intersection $J$ as follows. For such $J$, there exists a flat $S L(2, \mathbb{R})$-vector bundle $\xi: E \rightarrow \Sigma_{g}$ with $\left\langle e(\xi),\left[\Sigma_{g}\right]\right\rangle=J$ (see [4]). Let $L$ be the 0 -section of $\xi$. Then, $[L]^{2}=J$. Next, consider a flat $\mathbb{R}^{3}$-bundle $\eta=\xi \oplus \varepsilon^{1}$ where $\varepsilon^{1}$ is the trivial flat real line bundle. Then take the associated flat $S^{2}$-bundle $\zeta$ which is the required one. The subhemisphere bundle $\zeta_{+}$(resp. $\left.\zeta_{-}\right)$of $\zeta$ which corresponds to the hemisphere $\{(x, y, z) \in$ $S^{2} ; z>0$ (resp. $\left.\left.z<0\right)\right\}$ is nothing but the original flat vector bundle $\xi$ (resp. with its orientation reversed) so that the 0 -section corresponding to $(0,0, \pm 1)$ determines a compact leaf $L_{ \pm}$with $\left[L_{ \pm}\right]^{2}= \pm J$. 
Remark. In the above construction, if we fix the base space $\Sigma_{g}$, the selfintersection of a compact leaf $L_{ \pm}$is bounded by $g-1$, because the compact leaf is diffeomorphic to the base space. If we want to increase the self-intersection of a compact leaf, we have to embed a compact leaf of higher genus.

One way to do that would be to realize a compact leaf $L$ as a finite orbit of the holonomy group of another compact leaf $L_{0}$. However, in such a way, the self-intersection vanishes, because $L$ and $L_{0}$ are disjoint and $[L]=k \cdot\left[L_{0}\right] \epsilon$ $H_{2}(M)$, where $k$ is the covering degree of $L \rightarrow L_{0}$.

Another way would be to realize a compact leaf as a finite orbit of the global holonomy of a foliated bundle. But again, this has little hope as illustrated in the next example.

Example 1.6. Let us consider a foliated $S^{p}$-product over a closed oriented $p$ manifold $X$, i.e., a product $S^{p}$-bundle $\pi: M=X \times S^{p} \rightarrow X$ which has a $p$-dimensional foliation $\mathscr{F}$ whose leaves are transverse to the fibres. Let $L$ be a compact leaf of $\mathscr{F}$, and $k$ be the degree of the covering $\varphi=\left.\pi\right|_{L}: L \rightarrow X$. If $k$ is greater than 1 , the self-intersection $[L]^{2}$ vanishes.

Proof. Let $\tilde{\pi}: \widetilde{M}=L \times S^{p} \rightarrow L$ be the pull-back of the foliated $S^{p}$-product $\pi$ by $\varphi$ so that $\widetilde{M}$ admits a foliation $\widetilde{\mathscr{F}}$ which is transverse to the fibres of $\tilde{\pi}$, i.e., $\widetilde{\mathscr{F}}=\tilde{\varphi}^{*} \mathscr{F}$ where $\tilde{\varphi}: \widetilde{M} \rightarrow M$ is the covering map induced between the total spaces. The inverse image $\tilde{\varphi}^{-1}(L)=\widetilde{L}$ is mapped onto $L$ by $\left.\tilde{\varphi}\right|_{\tilde{L}}$ with degree $k$.

$$
\begin{aligned}
\widetilde{L}=L_{0} \cup L^{\prime} \subset \widetilde{M} & \stackrel{\tilde{\varphi}}{\longrightarrow} M \supset L \\
& \left.M \quad \begin{array}{ll}
\tilde{\pi} &
\end{array}\right\rfloor \quad(\varphi \text { degree } k) \\
& \stackrel{\varphi}{\longrightarrow} X
\end{aligned}
$$

The pull-back $\tilde{\pi}$ has the tautological section whose image $L_{0}$ is a connected component of $\widetilde{L}$. Therefore $\widetilde{L}$ splits into a disjoint union of $L_{0}$ and $L^{\prime}=$ $\widetilde{L} \backslash L_{0}$. They are mapped onto $L$ by $\tilde{\varphi}$ in degree 1 and $(k-1)$ respectively. The $p$ th homology groups and the induced homomorphisms corresponding to the above diagram constitute the following commutative diagram.

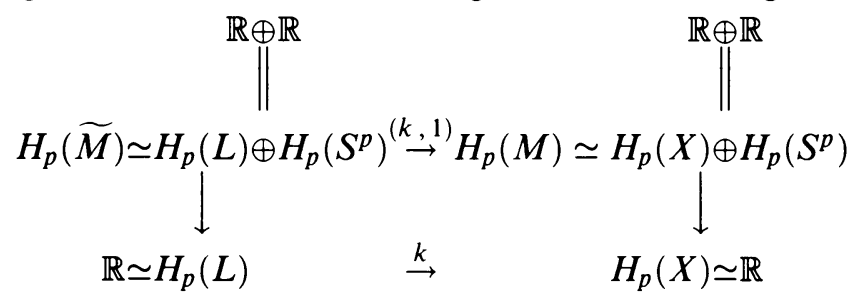

Therefore $\left[L^{\prime}\right]=(k-1)\left[L_{0}\right] \in H_{p}(\widetilde{M})$ is obtained and we see

$$
[L]^{2}=\left[L_{0}\right]^{2}=(k-1)^{-1}\left[L^{\prime}\right] \cdot\left[L_{0}\right] .
$$

As seen above, $L_{0}$ and $L^{\prime}$ are disjoint and thus $[L]^{2}=0$ is obtained.

These observations lead us to the following problem.

Problem. For a given $2 p$-dimensional manifold $M$, does there exist the upper bound for the self-intersection of any compact leaf of any $p$-dimensional foliation on $M$ ? How about in the case of foliated bundles with their base space and fibre specified? 


\section{VANISHING OF SELF-INTERSECTIONS}

2.1. In the previous section we observed an extreme class of foliation cycles and a phenomenon that cycles with larger supports tend to have the trivial selfintersections. In this section, we state and prove a vanishing theorem of the self-intersections for another extreme class of foliation cycles.

Theorem 2.1. Let $\mu$ be a transverse invariant measure of a foliated manifold $(M, \mathscr{F})$ supported on a smooth submanifold $N$ of $M$ satisfying the following conditions.

(1) $\operatorname{dim} N>p=\operatorname{dim} \mathscr{F}$.

(2) $\mu$ is bounded on $N$, i.e., for any transverse manifold $T$ of $\left(N,\left.\mathscr{F}\right|_{N}\right)$, the induced measure $\left.\mu\right|_{T}$ on $T$ is bounded w.r.t. any Riemannian volume of $T$.

Then the self-intersection of $C_{\mu}$ vanishes, i.e., $\left[C_{\mu}\right]^{2}=0$.

2.2. To prove Theorem 2.1, we construct a closed smooth $q$-form $\Phi_{\mu}=$ $\Phi\left(C_{\mu}, \varepsilon\right)$ which is the Poincare dual to the foliation cycle $C_{\mu}$ and show that $\left[\Phi_{\mu}\right]^{2}=0 \in H^{2 q}(M)$.

Let us first consider the case of a compact leaf $L$ and $C_{\mu}=\int_{L}$. We fix a Riemannian metric $g$ on $M$. Hence we consider that the tangent bundle $T M$ of $M$ splits as $T M=\tau \mathscr{F} \oplus \nu \mathscr{F}$ and $\nu \mathscr{F}=\tau \mathscr{F} \perp$ carries a fibre metric naturally. Let $E$ denote the total space of $\nu \mathscr{F}$ and $E_{r}$ denote the corresponding disc bundle $\{v \in E ;\|v\| \leq r\}$ of radius $r$. The Thom isomorphism implies that there exists a closed $q$-form $\Phi \in \Omega^{q}\left(E_{1}, \partial E_{1}\right)$ such that $\int_{E_{1, z}} \Phi=1$ over any point $z \in M$. We fix such a Thom form $\Phi$. Next, we choose $\varepsilon>0$ so that the exponential map

$$
\exp _{\varepsilon}:\left.E_{\varepsilon}\right|_{L} \rightarrow M
$$

is an embedding. Let $\mathbf{m}_{r}$ denote the fibrewise multiplication by $r$ on $E$, i.e.,

$$
\mathbf{m}_{r}: E \rightarrow E, \quad \mathbf{m}_{r}(z, v)=(z, r \cdot v) .
$$

Then, as is well known, the smooth $q$-form $\Phi(L, \varepsilon)=\left(\exp _{\varepsilon} \circ \mathbf{m}_{\varepsilon}\right)_{*} \Phi$ represents the Poincare dual to $[L]$.

2.3. To obtain the Poincare dual to foliation cycles $C_{\mu}$ in general, what we want to do is " $\int_{\mathscr{F}}$ [the above construction] $d \mu$." We fix a finite open covering $\mathscr{U}=\left\{U_{i}=D_{i}^{p} \times D_{i}^{q} ; i \in \mathscr{I}\right\}$ of $M$ by foliated charts and $\left\{\varphi_{i} ; i \in \mathscr{I}\right\}$ be a smooth partition of unity which is subordinate to the covering $\mathscr{U}$. We take $\varepsilon_{0}>0$ so small that $\exp _{\varepsilon_{0}}$ is an embedding over each plaque of $\mathscr{U}$. Now let $\Phi_{\varepsilon}$ denote the $q$-form $\left(\mathbf{m}_{\varepsilon}\right)_{*} \Phi$ on $E_{\varepsilon}$ which is also a Thom form and is concentrated on $E_{\varepsilon} . \Phi_{\varepsilon}$ is pushed down to $M$ leaf by leaf and is integrated along $\mathscr{F}$ (= the leaf space) by the measure $\mu$. To be precise, for each plaque $D_{i}^{p} \times\{y\}$ of $U_{i} \quad\left(y \in D_{i}^{q}\right)$, define a smooth $q$-form $\Phi(y, i, \varepsilon)$ on $M$ as

$$
\Phi(y, i, \varepsilon)=\left(\left.\exp _{\varepsilon}\right|_{D_{i}^{p} \times\{y\}}\right)_{*}\left(\varphi_{i} \circ \pi\right) \cdot \Phi_{\varepsilon}
$$

where $\pi$ denotes the bundle projection $E \rightarrow M$. Next, for each $U_{i}$ a smooth $q$-form $\Phi(i, \varepsilon)$ is defined at each point $z \in M$ as

$$
\Phi(i, \varepsilon)(z)=\int_{y \in D_{i}^{q}} \Phi(y, i, \varepsilon)(z) d \mu_{i}(y)
$$


where $\mu_{i}$ is the measure on $D_{i}^{q}$ induced from $\mu$. Finally, we set

$$
\Phi\left(C_{\mu}, \varepsilon\right)=\sum_{i \in \mathscr{I}} \Phi(i, \varepsilon)
$$

Proposition 2.2. (1) The q-form $\Phi\left(C_{\mu}, \varepsilon\right)$ is closed.

(2) The cohomology class of $\Phi\left(C_{\mu}, \varepsilon\right)$ does not depend on $\varepsilon$.

(3) The closed q-form $\Phi\left(C_{\mu}, \varepsilon\right)$ represents the Poincaré dual to the foliation cycle $C_{\mu}$.

Proof. (1) We first remark that the form $\Phi\left(C_{\mu}, \varepsilon\right)$ does not depend either on the choice of the covering $\mathscr{U}$ or on that of the partition of unity $\left\{\varphi_{i}\right\}$. Then, on a small neighbourhood of each point, the form $\Phi\left(C_{\mu}, \varepsilon\right)$ is considered to be the integral of closed forms on the neighbourhood along the transverse space by the measure $\mu$. Therefore $\Phi\left(C_{\mu}, \varepsilon\right)$ is closed.

(2) For $0<\delta<\varepsilon, \Phi_{\delta}$ and $\Phi_{\varepsilon}$ are cohomologuous in $E_{\varepsilon}$. Hence there exists a $(q-1)$-form $\Psi \in \Omega^{q-1}\left(E_{\varepsilon}, \partial E_{\varepsilon}\right)$ such that $d \Psi=\Phi_{\varepsilon}-\Phi_{\delta}$. Replacing $\Phi_{\varepsilon}$ by $\Psi$ in the above construction, we obtain a smooth $(q-1)$-form $\Psi\left(C_{\mu}\right) \in$ $\Omega^{q-1}(M)$ instead of $\Phi\left(C_{\mu}, \varepsilon\right)$. Then it is easy to see that $d \Psi\left(C_{\mu}\right)=\Phi\left(C_{\mu}, \varepsilon\right)-$ $\Phi\left(C_{\mu}, \delta\right)$.

(3) We show that the $p$-current

$$
C(\varepsilon)=\int_{M} \cdot \wedge \Phi\left(C_{\mu}, \varepsilon\right)
$$

(weakly) converges to the current $C_{\mu}$ as $\varepsilon \rightarrow 0$. Because the smooth form $\Phi\left(C_{\mu}, \varepsilon\right)$ is closed and its cohomology class is independent of $\varepsilon$, the same holds for $C(\varepsilon)$ and its homology class. Then, especially for any closed $p$-form $\omega \in \Omega^{p}(M)$, we have

$$
\langle\omega, C(\varepsilon)\rangle=\left\langle\omega, C_{\mu}\right\rangle \text { and }[C(\varepsilon)]=\left[C_{\mu}\right] \in H_{p}(M) .
$$

Therefore, it suffices to show the convergence.

By the construction of $\Phi\left(C_{\mu}, \varepsilon\right)$, it is enough to show that

$$
\int_{M} \omega \wedge \Phi(i, \varepsilon)=\int_{M} \omega \wedge\left(\int_{y \in D_{i}^{q}}\left(\left(\left.\exp _{\varepsilon}\right|_{D_{i}^{p} \times\{y\}}\right)_{*}\left(\varphi_{i} \circ \pi\right) \cdot \Phi_{\varepsilon}\right) d \mu_{i}(y)\right)
$$

converges to $\left\langle\varphi_{i} \cdot \omega, C(\varepsilon)\right\rangle=\left\langle\omega, \varphi_{i} \cdot C_{\mu}\right\rangle$ as $\varepsilon \rightarrow 0$. By Fubini's theorem, we have

$$
\int_{M} \omega \wedge \Phi(i, \varepsilon)=\int_{y \in D_{i}^{q}}\left(\int_{M} \omega \wedge\left(\left.\exp _{\varepsilon}\right|_{D_{i}^{p} \times\{y\}}\right)_{*}\left(\varphi_{i} \circ \pi\right) \cdot \Phi_{\varepsilon}\right) d \mu_{i}(y) .
$$

Now, we look at the integrand

$$
\begin{aligned}
F_{\varepsilon}(y) & =\int_{M} \omega \wedge\left(\left.\exp _{\varepsilon}\right|_{D_{i}^{p} \times\{y\}}\right)_{*}\left(\varphi_{i} \circ \pi\right) \cdot \Phi_{\varepsilon} \\
& =\int_{E_{1}}\left(\left.\exp _{\varepsilon}\right|_{D_{i}^{p} \times\{y\}} \circ \mathbf{m}_{\varepsilon}\right)^{*} \omega \wedge\left(\varphi_{i} \circ \pi\right) \cdot \Phi
\end{aligned}
$$

on $D_{i}^{q}$. In the second expression of $F_{\varepsilon}(y)$, the norm of $\left(\left.\exp _{\varepsilon}\right|_{D_{i}^{p} \times\{y\}} \circ \mathbf{m}_{\varepsilon}\right)^{*}$ is bounded as $\varepsilon \rightarrow 0$. Therefore the function $F_{\varepsilon}(y)$ is bounded on $\left(0, \varepsilon_{0}\right) \times D_{i}^{q}$. Moreover, it is clear that the form $\left(\left.\exp _{\varepsilon}\right|_{D_{i}^{p} \times\{y\}} \circ \mathbf{m}_{\varepsilon}\right)^{*} \omega$ on $E_{1}$ uniformly 
converges to $\left(\left.\pi\right|_{D_{i}^{p} \times\{y\}}\right)^{*} \omega$ as $\varepsilon \rightarrow 0$. Then, the limit $F(y)=\lim _{\varepsilon \rightarrow 0} F_{\varepsilon}(y)$ is obtained as

$$
\begin{aligned}
F(y) & =\int_{E_{1}}\left(\left.\pi\right|_{D_{i}^{p} \times\{y\}}\right)^{*} \omega \wedge\left(\varphi_{i} \circ \pi\right) \cdot \Phi \\
& =\int_{E_{1}}\left(\left.\pi\right|_{D_{i}^{p} \times\{y\}}\right)^{*}\left(\varphi_{i} \cdot \omega\right) \wedge \Phi \\
& =\int_{D_{i}^{p} \times\{y\}} \varphi_{i} \cdot \omega
\end{aligned}
$$

and turns out to be bounded and continuous on $D_{i}^{q}$. Therefore, by the bounded convergence theorem, we obtain

$$
\begin{aligned}
\lim _{\varepsilon \rightarrow 0} \int_{M} \omega \wedge \Phi(i, \varepsilon)=\lim _{\varepsilon \rightarrow 0} \int_{y \in D_{i}^{q}} F_{\varepsilon}(y) d \mu_{i}(y) \\
\quad=\int_{y \in D_{i}^{q}} \lim _{\varepsilon \rightarrow 0} F_{\varepsilon}(y) d \mu_{i}(y) \\
\quad=\int_{y \in D_{i}^{q}}\left(\int_{D_{i}^{p} \times\{y\}} \varphi_{i} \cdot \omega\right) d \mu_{i}(y)=\left\langle\varphi_{i} \cdot \omega, C_{\mu}\right\rangle
\end{aligned}
$$

and thus the proof is completed.

2.4. Now, let us prove Theorem 2.1. We may assume $M=N$ because, if not so, we only have to replace $(M, \mathscr{F})$ by $\left(N,\left.\mathscr{F}\right|_{N}\right)$. What we actually prove is the uniform convergence

$$
\lim _{\varepsilon \rightarrow 0} \Phi\left(C_{\mu}, \varepsilon\right) \wedge \Phi\left(C_{\mu}, \varepsilon\right)=0
$$

For this, we have to know the behaviour of $\Phi\left(C_{\mu}, \varepsilon\right)$ as $\varepsilon \rightarrow 0$ and of the exponential map $\exp _{\varepsilon}$ a little more closely.

For any point $z \in M$, choose a foliated chart $U=D^{p} \times D^{q}=\{(x, y)$; $\left.x=\left(x_{1}, \ldots, x_{p}\right), y=\left(y_{1}, \ldots, y_{q}\right)\right\}$ around $z$ as follows. First, let $z$ be the origin $(0,0)$ and $L$ be the leaf through $z$. Take orthonormal framings $\left(V_{1}, \ldots, V_{p}\right)$ and $\left(W_{1}, \ldots, W_{q}\right)$ of $\left.\tau \mathscr{F}\right|_{L}$ and $\left.\nu \mathscr{F}\right|_{L}$ respectively around $z$ and take the normal coordinate $\left(x_{1}, \ldots, x_{p}\right)$ of $L$ around $z$ w.r.t. the framing $\left(V_{1}(z), \ldots, V_{p}(z)\right)$ and the metric $\left.\mathfrak{g}\right|_{L}$. Then, we define the coordinate $\left(\xi=\left(\xi_{1}, \ldots, \xi_{p}\right), \eta=\left(\eta_{1}, \ldots, \eta_{q}\right)\right)$ of $\left.E\right|_{L}$ around $z$ as $(\xi, \eta)=$ $\left(x, \eta_{1} \cdot W_{1}(x, 0)+\cdots+\eta_{q} \cdot W_{q}(x, 0)\right)$. Next, take the normal coordinate $\left(y_{1}\right.$, $\left.\ldots, y_{q}\right)$ of the transverse disk $\exp _{\varepsilon_{0}}\left(E_{\varepsilon_{0}, z}\right)$ w.r.t. the framing $\left(W_{1}(z), \ldots\right.$, $\left.W_{q}(z)\right)$ and the metric $\mathfrak{g}$, and thus as a foliated chart, the $y$-coordinate is defined around $z$. At $(0, y)$, the framing $\exp _{*(0, y=\eta)}\left(\left(V_{1}, \ldots, V_{p}\right)\right)=$ $\left(V_{1}^{\prime}, \ldots, V_{p}^{\prime}\right)$ may not be orthonormal. Therefore, by the orthogonal projection, $\left(V_{1}^{\prime}, \ldots, V_{p}^{\prime}\right)$ is projected to the framing $\left(V_{1}^{\prime \prime}, \ldots, V_{p}^{\prime \prime}\right)$ of $\tau \mathscr{F}(0, y)$ and, by the Gram-Schmidt method, we obtain an orthonormal framing $\left(V_{1}, \ldots, V_{p}\right)$ of $\tau \mathscr{F}_{(0, y)}$. Then, take the normal coordinate $\left(x_{1}, \ldots, x_{p}\right)$ on the leaf $L_{y}$ through $(0, y)$ around $(0, y)$ w.r.t. the framing $\left(V_{1}, \ldots, V_{p}\right)_{(0, y)}$ and the metric $\left.\mathfrak{g}\right|_{L_{y}}$. Thus we obtain the foliated chart $\{(x, y)\}=D^{p} \times D^{q}=U$ of some radii $\varepsilon_{1}$ and $\varepsilon_{2}$ of $D^{p}$ and $D^{q}$ respectively. The smoothness of $(M, \mathscr{F})$ and the compactness of $M$ guarantees that we can choose $\varepsilon_{1}$ and $\varepsilon_{2}$ uniformly, i.e., they do not depend on $z$ but only on $(M, \mathscr{F})$ and $\mathfrak{g}$. We also obtain 
an orthonormal framing $\left(V_{1}, \ldots, V_{p}, W_{1}, \ldots, W_{q}\right)$ of $\left.\tau \mathscr{F} \oplus \nu \mathscr{F}\right|_{U}=\left.T M\right|_{U}$ from the framing $\left(\frac{\partial}{\partial x_{1}}, \ldots, \frac{\partial}{\partial x_{p}}, \frac{\partial}{\partial y_{1}}, \ldots, \frac{\partial}{\partial y_{q}}\right)$ by the Gram-Schmidt method.

Then, by the coordinates $(\xi, \eta)$ and $(x, y)$, the exponential map

$$
\mathrm{E}=\exp _{\varepsilon_{2}}:\left.E_{\varepsilon_{2}}\right|_{D^{p} \times\{0\}} \rightarrow U=D^{p} \times D^{q}
$$

is expressed as $x_{i}=\xi_{i}+f_{i}(\xi, \eta)$ and

$$
y_{i}=\sum_{l=1}^{q}\left(\delta_{j l}+a_{j l}(\xi)\right) \cdot \eta_{l}+g_{j}(\xi, \eta)
$$

where $\delta_{j l}$ denotes Kronecker's symbol, with the estimates

$$
f_{i}(\xi, \eta) \in O(\|\xi\|) \cdot O(\|\eta\|), \quad g_{j}(\xi, \eta) \in O(\|\xi\|) \cdot O\left(\|\eta\|^{2}\right),
$$

and $a_{j l}(\xi) \in O(\|\xi\|)$.

Therefore its differential $D E$ is expressed around $z$ as

$$
D \mathrm{E}=\left(\begin{array}{cc}
\delta_{i k}+f_{i k}(\xi, \eta), & f_{i l}(\xi, \eta) \\
\sum_{l} a_{j l k}(\xi) \cdot \eta_{l}+g_{j k}(\xi, \eta), & \delta_{j l}+a_{j l}(\xi)+g_{j l}(\xi, \eta)
\end{array}\right)
$$

with the estimates

$$
\begin{gathered}
f_{i k}(\xi, \eta) \in O(\|\eta\|), \quad f_{i l}(\xi, \eta) \in O(\|\xi\|), \\
a_{j \rho k}(\xi) \in O(1), \quad g_{j k}(\xi, \eta) \in O\left(\|\eta\|^{2}\right),
\end{gathered}
$$

and

$$
g_{j l}(\xi, \eta) \in O(\|\xi\|) \cdot O(\|\eta\|) .
$$

Also, we remark that the framing $\left(V_{1}, \ldots, V_{p}\right)$ (resp. $\left.\left(W_{1}, \ldots, W_{q}\right)\right)$ coincides with $\left(\frac{\partial}{\partial x_{1}}, \ldots, \frac{\partial}{\partial x_{p}}\right)$ (resp. $\left.\left(\frac{\partial}{\partial y_{1}}, \ldots, \frac{\partial}{\partial y_{q}}\right)\right)$ at $z=(0,0)$ and both of them span the same tangent plane $\tau \mathscr{F}$ (resp. $\nu \mathscr{F}$ ) along $D^{p} \times\{0\}$, i.e., if we express the second by the linear combination of the first as

$$
\frac{\partial}{\partial x_{i}}=\sum_{k}\left(\delta_{i k}+b_{i k}(x, y)\right) \cdot V_{k}+\sum_{l} b_{i l}^{\prime}(x, y) \cdot W_{l}
$$

and

$$
\frac{\partial}{\partial y_{j}}=\sum_{k} b_{j k}(x, y) \cdot V_{k}+\sum_{l}\left(\delta_{j l}+b_{i l}^{\prime}(x, y)\right) \cdot W_{l},
$$

we have the estimates that the functions $b_{i k}$ and $b_{j l}^{\prime}$ belong to $O(\|(x, y)\|)$ and $b_{i l}^{\prime}$ and $b_{j k}$ belong to $O(\|y\|)$. Of course these functions depend on the choice of the center $z=(0,0)$ and the orthonormal framing there. But the smoothness and the compactness imply that there exists a constant $K_{1}>0$ which depends only on $(M, \mathscr{F})$ and $\mathfrak{g}$ such that

$$
\begin{gathered}
\left|f_{i k}\right|,\left|a_{j l k}\right|,\left|b_{i l}^{\prime}\right|,\left|b_{j k}\right| \leq K_{1} \cdot\|\eta\|, \quad\left|f_{i l}\right|,\left|a_{j l}\right| \leq K_{1} \cdot\|\xi\|, \\
\left|g_{j k}\right| \leq K_{1} \cdot\|\eta\|^{2}, \quad\left|g_{j l}\right| \leq K_{1} \cdot\|\xi\| \cdot\|\eta\|,
\end{gathered}
$$

and

$$
\left|b_{i k}\right|,\left|b_{j l}^{\prime}\right| \leq K_{1} \cdot\|(x, y)\|
$$

and $\eta$ on the r.h.s. can be replaced by $y$ through the map E. 
Next, we estimate the form $\left.\Phi_{\varepsilon}\right|_{D^{p} \times\{0\}}$ as $\varepsilon \rightarrow 0$. On $\left.E_{\varepsilon_{2}}\right|_{D^{p} \times\{0\}}$, using the coordinate $(\xi, \eta)$, the $q$-form $\left.\Phi\right|_{D^{p} \times\{0\}}$ is expressed as

$$
\Phi_{1}=\left.\Phi\right|_{D^{p} \times\{0\}}=\sum_{s=0}^{q} \sum_{\substack{|I|=s \\|J|=q-s}} \alpha_{I J}(\xi, \eta) d \xi_{i_{1}} \wedge \cdots \wedge d \xi_{i_{s}} \wedge d \eta_{j_{i}} \wedge \cdots \wedge d \eta_{j_{q-s}} .
$$

Here, $I=\left(i_{1}, \ldots, i_{s}\right)$ and $J=\left(j_{1}, \ldots, j_{q-s}\right)$ are multi-indices such that

$$
1 \leq i_{1}<\cdots<i_{s} \leq p \text { and } 1 \leq j_{1}<\cdots<j_{q-s} \leq q
$$

and $|I|$ and $|J|$ denote their length. Here again, there exists a constant $K_{2}$ which depends only on $(M, \mathscr{F})$ and $\mathfrak{g}$ such that

$$
\left|\alpha_{I J}(\xi, \eta)\right| \leq K_{2} \text {. }
$$

As the multiplication map $\mathbf{m}_{\varepsilon}$ is simply expressed as $\mathbf{m}_{\varepsilon}(\xi, \eta)=(\xi, \varepsilon \eta)$, we obtain

$$
\Phi_{\varepsilon}(\xi, \eta)=\sum_{s} \sum_{I, J} \varepsilon^{-|J|} \alpha_{I J}\left(\xi, \varepsilon^{-1} \eta\right) d \xi_{i_{1}} \wedge \cdots \wedge d \xi_{i_{s}} \wedge d \eta_{j_{1}} \wedge \cdots \wedge d \eta_{j_{q-s}} .
$$

As our estimate is uniform w.r.t. $z$, it is enough to estimate the form $\mathrm{E}_{*} \Phi_{\varepsilon}$ only on the transverse disk $\{x=0\}=\mathrm{E}(\{\xi=0\})$. Therefore we may assume $f_{i l}=0$. Combining all above estimates and this remark, we obtain the following estimate of the form $\mathrm{E}_{*} \Phi_{\varepsilon}$.

$$
\begin{aligned}
\mathrm{E}_{*} \boldsymbol{\Phi}_{\varepsilon}= & \sum_{s} \sum_{I, J} \alpha_{I J} \varepsilon^{-|J|} \bigwedge_{i \in I}\left(d x_{i}+\sum_{k=1}^{p} h_{I i k} d x_{k}+\sum_{l=1}^{q} c_{I i l} d y_{l}\right) \\
& \wedge \bigwedge_{j \in J}\left(\sum_{l=1}^{q} t_{J j l} d y_{l}\right) .
\end{aligned}
$$

Here, $h_{I i k}, c_{I i l}$, and $t_{J j l}$ are some smooth functions such that $h_{I i k} \in O(\|y\|)$, and $c_{I i l}, t_{J j l} \in O(1)$. Putting the above in order by $d x_{i}$ 's and $d y_{j}$ 's, we obtain

$$
\mathrm{E}_{*} \boldsymbol{\Phi}_{\varepsilon}=\sum_{s} \sum_{I, J} \psi_{I J} d x_{i_{1}} \wedge \cdots \wedge d x_{i_{s}} \wedge d y_{j_{1}} \wedge \cdots \wedge d y_{j_{q-s}}
$$

where $\psi_{I J}$ is some function belonging to $O\left(\varepsilon^{-|J|}\right)$, i.e., there exists some constant $K_{3}$ which depends only on $(M, \mathscr{F})$ and $\mathfrak{g}$ such that $\left|\psi_{I J}(0, y)\right| \leq$ $K_{3} \cdot \varepsilon^{-|J|}$. If we use the dual basis of 1 -forms $\left(\alpha_{1}, \ldots, \alpha_{p}, \beta_{1}, \ldots, \beta_{q}\right)$ to the framing $\left(V_{1}, \ldots, V_{p}, W_{1}, \ldots, W_{q}\right)$, we obtain the similar estimate

$$
\mathrm{E}_{*} \Phi_{\varepsilon}=\sum_{s} \sum_{I, J} \varphi_{I J} \alpha_{i_{1}} \wedge \cdots \wedge \alpha_{i_{s}} \wedge \beta_{j_{1}} \wedge \cdots \wedge \beta_{j_{q-s}}
$$

where $\varphi_{I J}$ 's satisfy the same condition as $\psi_{I J}$ 's. Here we introduce the notation

$$
\alpha_{I}=\alpha_{i_{1}} \wedge \cdots \wedge \alpha_{i_{s}} \text { and } \beta_{J}=\beta_{j_{1}} \wedge \cdots \wedge \beta_{j_{q-s}} .
$$

The final estimate is given as follows. To obtain the estimate at a point $m \in M$, take a foliated chart $U=D^{p} \times D^{q}=\{(x, y)\}$ around $m$. For a point $z=(x, y)$ we obtain the above $q$-form $\mathrm{E}_{*} \Phi_{\varepsilon}$ which is now denoted by $\Phi(y, \varepsilon)$. We may assume that there exists a constant $K_{4}>0$ which depends only on $(M, \mathscr{F})$ and $\mathfrak{g}$ such that if $\|y\|>K_{4} \cdot \varepsilon$ the point $m=(0,0)$ is not 
in the support of $\Phi(y, \varepsilon)$. Thus the Poincaré dual $\Phi\left(C_{\mu}, \varepsilon\right)$ to $C_{\mu}$ around $m$ is given by

$$
\Phi\left(C_{\mu}, \varepsilon\right)=\int_{y \in D\left(K_{4} \cdot \varepsilon\right)} \Phi(y, \varepsilon) d \mu(y)
$$

where $D(r)$ denotes the subset $\{y ;\|y\| \leq r\}$ of $D^{q}$ and $d \mu$ is the measure on $D^{q}$ determined by $\mu$. Therefore around $m$, we obtain

$$
\begin{gathered}
\Phi\left(C_{\mu}, \varepsilon\right) \wedge \Phi\left(C_{\mu}, \varepsilon\right) \\
=\iint_{\left(y_{1}, y_{2}\right) \in D\left(K_{4} \cdot \varepsilon\right)^{2}} \Phi\left(y_{1}, \varepsilon\right) \wedge \Phi\left(y_{2}, \varepsilon\right) d \mu \times d \mu\left(y_{1}, y_{2}\right) \\
=\iint_{\left(y_{1}, y_{2}\right) \in D\left(K_{4} \cdot \varepsilon\right)^{2}}\left(\sum_{s_{1}} \sum_{I_{1}, J_{1}} \varphi_{I_{1} J_{1}} \alpha_{I_{1}} \wedge \beta_{J_{1}}\right) \\
\wedge\left(\sum_{s_{2}} \sum_{I_{2}, J_{2}} \varphi_{I_{2} J_{2}} \alpha_{I_{2}} \wedge \beta_{J_{2}}\right) d \mu \times d \mu\left(y_{1}, y_{2}\right) \\
=\iint_{\left(y_{1}, y_{2}\right) \in D\left(K_{4} \cdot \varepsilon\right)^{2}} \sum_{s_{1}, s_{2}} \sum_{I_{1}, J_{1}, I_{2}, J_{2}} \varphi_{I_{1} J_{1}} \varphi_{I_{2} J \cdot 2} \alpha_{I_{1}} \wedge \beta_{J_{1}} \wedge \alpha_{I_{2}} \wedge \beta_{J_{2}} d \mu \times d \mu\left(y_{1}, y_{2}\right) .
\end{gathered}
$$

If $\left|J_{1}\right|+\left|J_{2}\right|>q, \beta_{J_{1}} \wedge \beta_{J_{2}}=0$. Therefore, putting the above in order, we obtain the following estimate

$$
\begin{aligned}
& \Phi\left(C_{\mu}, \varepsilon\right) \wedge \Phi\left(C_{\mu}, \varepsilon\right) \\
&=\iint_{\left(y_{1}, y_{2}\right) \in D\left(K_{4} \cdot \varepsilon\right)^{2}} \sum_{s} \sum_{I, J} \Phi_{I J} \cdot \alpha_{I} \wedge \beta_{J} d \mu \times d \mu\left(y_{1}, y_{2}\right) .
\end{aligned}
$$

Here, we may assume that there exists a constant $K_{5}$ which depends only on $(M, \mathscr{F})$ and $\mathfrak{g}$ such that the functions $\Phi_{I J}$ are estimated as

$$
\left|\Phi_{I J}\right| \leq K_{5} \cdot \varepsilon^{-|J|}
$$

Therefore, there exists a constant $K_{6}$ of the same kind such that the integrand of the above formula is estimated as

$$
\left\|\sum_{s} \sum_{I, J} \Phi_{I J} \cdot \alpha_{I} \wedge \beta_{J}\right\| \leq K_{6} \cdot \varepsilon^{-q}
$$

where the 1.h.s. is the natural norm as a $q$-form. On the other hand, the assumption that $\mu$ is bounded against the Lebesgue measure implies that there exists a constant $K_{7}$ of the same kind such that $d \mu \times d \mu(D(r) \times D(r))$ is estimated as

$$
d \mu \times d \mu(D(r) \times D(r)) \leq K_{7} \cdot r^{2 q} .
$$

Combining the last two estimates, we obtain the uniform estimate

$$
\left\|\Phi\left(C_{\mu}, \varepsilon\right) \wedge \Phi\left(C_{\mu}, \varepsilon\right)\right\| \leq K_{4}^{2 q} \cdot K_{6} \cdot K_{7} \cdot \varepsilon^{q}
$$

and thus the uniform convergence

$$
\lim _{\varepsilon \rightarrow 0}\left\|\Phi\left(C_{\mu}, \varepsilon\right) \wedge \Phi\left(C_{\mu}, \varepsilon\right)\right\|=0
$$

is obtained. This completes the proof of Theorem 2.1 . 
Example 2.3. Let $(M, \mathscr{F}, \mathfrak{h})$ be a Riemannian foliation with a holonomy invariant transverse Riemannian metric $\mathfrak{h}$. Then $\mathfrak{h}$ defines a smooth transverse invariant volume $\mu$ and $\left[C_{\mu}\right]^{2}$ vanishes.

\section{REFERENCES}

1. R. Bott, Lectures on characteristic classes of foliations, Lecture Notes in Math., vol. 279, Springer-Verlag, 1972.

2. M. Gromov, Volume and bounded cohomology, Publ. Math. Inst. Hautes Études Sci. 56 (1982), 5-100.

3. S. Hurder and Y. Mitsumatsu (in preparation).

4. J. W. Milnor, On the existence of a connection with curvature zero, Comment. Math. Helv. 32 (1958), 215-223.

5. Y. Mitsumatsu, Self-intersections and transverse euler numbers of foliation cycles, Thesis, University of Tokyo, 1985.

6. D. Sullivan, A generalization of Milnor's inequality concerning affine foliations and affine manifolds, Comment. Math. Helv. 51 (1976), 183-189.

7. _ Cycles for the dynamical study of foliated manifolds and complex manifolds, Invent. Math. 36 (1976), 225-255.

Department of Mathematics, Faculty of Science and Engineering, Chuo University, 1-13-27 Kasuga, Bunkyo-KU, TOKYo 112, JaPAN

E-mail address: yoshi@indsys.chuo-u.ac.jp 